\title{
CLÁUSULA REBUS SIC STANTIBUS: ¿UNA OPCIÓN PARA EL PROBLEMA DEL AGUA EN LA FRONTERA NORTE?
}

\author{
Nora RAMÍREZ FLORES*
}

\begin{abstract}
RESUMEN: Una de las doctrinas del derecho internacional más polémicas a lo largo de la historia es la llamada Cláusula Rebus Sic Stantibus. El artículo hace un recuento histórico de la evolución de está cláusula, acercándola a la posibilidad de su invocación para resolver el conflicto bilateral que se ha generado alrededor del comúnmente denominado Tratado de Aguas Internacionales entre México y Estados Unidos de América. Las obligaciones de suministro de agua que debe cubrir México se han vuelto materia de polémica, en virtud de las graves sequías que han afectado a nuestro país y las actas que se han firmado para garantizar el cumplimiento. Estos dos factores implican, para la autora, un cambio fundamental en las condiciones que dieron origen al tratado.
\end{abstract}

ABSTRACT: Through history, the Rebus Sic Stantibus Clause has been one of the most polemic theories of International Law. This article presents a brief history of its evolution, in order to bring about the possibility of implementing it to solve the bilateral conflict created by the international waters treaty signed by Mexico and the U. S. The Mexican obligation to provide water to the U. S. has become a polemic topic given the fact of both, the intense droughts that our country has recently suffered, and of the undertaken compromises to ensure its compliance. The current situation implies for the author a fundamental change in the circumstances that originated the treaty.

RÉSUMÉ: Une des doctrines du Droit International le plus polémiques, historiquement, est la Clause Rebus Sic Stantibus. Cet article réalise un historique de l'évolution de cette clause ainsi que étudie la possibilité de faire appel à celle-ci pour résoudre le conflit bilatéral qui existe autour du Traité de Eaux Internationaux entre le Mexique et les Etats-Unis d'Amérique. Les obligations du Mexique en tant que fournisseur d'eau sont devenus un sujet polémique à cause des multiples sécheresses que le Mexique a souffert, mettant en cause les Traités que le Mexique a signé pour garantir l'accomplissement de ses obligations. Pour l'auteur, ceci implique des changements fondamentaux des conditions qui ont donné lieu au Traité.

* Académica de la Facultad de Derecho y maestra de derecho internacional público. 
SUMARIO: I. Introducción. II. Cláusula Rebus Sic Stantibus. III. Convención de Viena sobre el Derecho de los Tratados, 1980. IV. La aplicación de la Cláusula Rebus Sic Stantibus. V. El Tratado sobre Distribución de Aguas Internacionales entre los Estados Unidos Mexicanos y los Estados Unidos de América. VI. Características del tratado. Particularidades del suministro. VII. La sequía y el conflicto internacional. VIII. Condiciones hidrológicas y cambio climático. Un cambio en las circunstancias. IX. Conclusión. Viabilidad de la aplicación de la Cláusula Rebus Sic Stantibus en el Tratado de Aguas Internacionales.

\section{INTRODUCCIÓN}

Desde los orígenes de los Estados, ha existido una indispensable necesidad de interrelación entre los mismos. La manera más usual y jurídicamente más aceptada para que las naciones cumplan y hagan cumplir sus derechos, obligaciones y acuerdos ante otros Estados es a través de la celebración de tratados internacionales.

Conforme fue avanzando la historia de las relaciones entre reinos, imperios y Estados, se han ido generando y perfeccionando mecanismos que norman y guían la formalización de acuerdos y la celebración de tratados. En la actualidad, el mecanismo internacional de mayor referencia al respecto, es la Convención de Viena sobre el Derecho de los Tratados, la cual entró en vigor en 1980.

Los tratados internacionales tienen como finalidad establecer las condiciones y normatividad para que las partes firmantes cumplan con un objetivo específico. Reseña puntualmente sus derechos y obligaciones, así como el tiempo de aplicación y posibles soluciones a situaciones que pudieran causar una controversia entre las partes. Sin embargo, la mayoría de los tratados no pueden prever todos los cambios o circunstancias que afectarán el objeto de los mismos a lo largo del tiempo. En muchas ocasiones, las circunstancias que dieron origen a un tratado o que rodean el entorno y los compromisos del mismo, pueden variar en forma tan profunda, que llega a perderse el objeto por el cual fue suscrito. En algunos otros casos, las partes quedan inclusive imposibilitadas para cumplir sus obligaciones, a pesar de que exista voluntad para hacerlo. La historia diplomática cuenta ya en su haber con interesantes casos en los 
que el cambio de la circunstancia fundamental que dio origen a la firma de un tratado se modifica a tal grado, que ha sido necesario llevar a cabo nuevos acuerdos para evitar conflictos internacionales, o inclusive, caducarlo (podemos citar, como ejemplo, el Tratado de París de 1856 o la suspensión del pago de la deuda francesa a Estados Unidos de América -EUA- en 1932). ${ }^{1}$

Las opiniones entre maestros y doctrinarios del derecho internacional se dividen respecto a la conveniencia o no de contar con una cláusula de corrección. Mientras que unos la consideran como un complemento necesario al clausulado de los tratados (Rebus Sic Stantibus), otros opinan que rompe con el espíritu mismo de la celebración de los acuerdos internacionales, ya que puede presuponer y autorizar la denuncia de los mismos. Para qué signamos un convenio si estamos predispuestos a romperlo. Como menciona Remiro Brotóns, desde que en 1612 Gentili afirmó que Omnis conventio intelligitur rebus sic stantibus, la significación del cambio en las circunstancias de la celebración del tratado para la vida de éste ha sido uno de los puntos más apasionadamente controvertidos en la historia del derecho internacional. ${ }^{2}$

Por otro lado, dentro de las relaciones diplomáticas y particularmente las relaciones bilaterales, una de las más dinámicas en el mundo es la que se lleva a cabo entre Estados Unidos Mexicanos y EUA. En esta relación, por naturaleza e historia complicada, existen multitud de acuerdos que permiten dar certidumbre al cotidiano proceso bilateral. Algunos de ellos son indispensables para evitar situaciones de conflicto por el uso, dominio y posesión de recursos valiosos para ambas naciones: particularmente, el agua. De esta manera, el presente artículo busca realizar un análisis sobre las condiciones generales que imperan actualmente en el Tratado sobre Distribución de Aguas Internacionales entre los Estados Unidos Mexicanos y los Estados Unidos de América, comúnmente conocido como el Tratado de Aguas Internacionales de 1944 y la posibilidad de encontrar una alternativa jurídica a la pesada carga que representa para nuestra nación, cumplir con los compromisos sobre la dotación de

1 Para abundar sobre estos ejemplos, puede consultarse a Gómez Robledo, Antonio, "La Cláusula Rebus Sic Stantibus", Estudios internacionales, México, Secretaría de Relaciones Exteriores, 1978.

2 Remiro Brotóns, Antonio, Derecho internacional público, t. II: Derecho de los tratados, Madrid, Tecnos, 1987, p. 484. 
agua que el tratado implica. Nos estamos refiriendo a la viabilidad de la aplicación de la Cláusula Rebus Sic Stantibus (RSS).

Durante la primera parte del artículo, proponemos un breve marco teórico-histórico sobre esta denominada "cláusula de escape". Más adelante describimos algunas de las particularidades del Tratado de Aguas Internacionales, las condiciones en que éste se firmó en 1944, los cambios que se han sucedido desde entonces, así como los principales conflictos que han surgido y sus propuestas de solución. Terminamos con algunas reflexiones sobre la posibilidad de apelar al arbitraje internacional para aliviar a México de la onerosa carga que representa el pago de grandes volúmenes de agua en los cada vez más prolongados periodos de sequía, apelando a la $R S S$.

\section{ClÁuSULA REBUS SIC STANTIBUS}

El maestro Galindo, en su Diccionario jurídico establece que Rebus Sic Stantibus es una expresión en latín que surge en la Edad Media por los canonistas. La expresión $R S S$ ha servido para denominar a una de las teorías jurídicas más importantes que buscan con deseo la equidad y la justicia, principios rectores de los sistemas jurídicos. ${ }^{3}$

La expresión latina de la cláusula puede entenderse como "estando así las cosas" o "manteniéndose en ese estado"; dándole una interpretación más contemporánea, podemos decir que dicha expresión significa que las circunstancias se sigan manteniendo en el estado que guardaban al momento de celebrarse el contrato o tratado, de tal forma que si varían las circunstancias que al momento prevalecían, se produciría una ventaja desproporcionada para una de las partes.

El maestro Antonio Gómez Robledo ${ }^{4}$ señala que esta cláusula tiene su formulación en la era cristiana y que no puede compararse ni a la doctrina de la equidad, ni tampoco a la laesio enormis del derecho romano, porque en ninguno de estos conceptos está implícita la idea fundamental que persigue la $R S S$ la cual, haciendo referencia a Bierly, implica lo siguiente:

3 Galindo Garfias, Ignacio, Diccionario jurídico mexicano, 12a. ed., México, Porrúa-UNAM, Instituto de Investigaciones Jurídicas, 1998, pp. 1637 y 1638.

4 Gómez Robledo, Antonio, op. cit., nota 1. 
Lo que pone fin a un tratado es la desaparición del fundamento en que descansa; o si preferimos ponerlo en términos subjetivos, el tratado termina en razón de que de sus términos podemos inferir que las partes, por más que no lo hayan dicho expresamente, habrían preferido que el tratado caducara, si hubieran podido prever el acontecimiento que de hecho ha sobrevenido. La cláusula, en suma, es una norma interpretativa que nos garantiza que habrá de darse siempre un efecto razonable al tratado, antes de que el efecto irracional que resultara de una adhesión literal a sus términos. ${ }^{5}$

De acuerdo con juristas como Raoul Genet, es Hugo Grocio el que introduce la cláusula en el derecho internacional moderno, al señalar que los acuerdos entre Estados suelen disputarse "si las promesas llevan consigo la condición tácita de que las cosas permanezcan en su estado actual. A esto habrá que responder negativamente, a no ser que sea del todo evidente que el presente estado de cosas estuvo contemplado en la razón única que llevó a concertar el tratado". ${ }^{6}$

Por su parte, Alberico Gentili lleva a cabo una precisión sobre la aplicación de la cláusula, al introducir en su doctrina un elemento adicional al del cambio fundamental de las circunstancias, al referir que cualquiera de las partes está dispensada de cumplir un tratado si las circunstancias fundamentales han cambiado "y si, además, no fue previsto este cambio".7

La introducción de este segundo elemento, la falta de previsión, junto con el cambio fundamental de las circunstancias que dieron origen a un tratado, completan el concepto de la cláusula $R S S$, al vincular la doctrina $R S S$, con la teoría de la imprevisión.

Sin embargo, no todos los doctrinarios estuvieron de acuerdo con la introducción de la cláusula en la doctrina del derecho internacional. Así, por ejemplo, Bynkershoek señala que $R S S$ "no es sino un expediente maquiavélico para eximirse de las obligaciones contraídas en los tratados, o de otro modo, la máscara de la ruptura de la fe jurada: Ruptae fidei velementum". ${ }^{8}$

5 Bierly, The Law of Nations, 6a. ed., Exford, 1963, p. 336.

6 Grocio, Hugo, De iure belli ac pacis, lib. II, cap. 16, párr. 25.

7 Gentilli, Alberico, De iure belli libri tres, lib. III, cap. XVI.

8 Bynkershoek, Quaestionum iuris publici libri duo, lib. II, cap. X. 
De acuerdo con el maestro Gómez Robledo, el último de los clásicos del derecho internacional, Emer de Batel, es un ardiente defensor de la cláusula, al expresar que:

Una cuestión que se propone y se discute es la de saber si las promesas encierran la condición tácita de que las cosa se mantengan en el estado en que están, de tal suerte que el cambio que sobrevenga en el estado de cosas pueda introducir una excepción en la promesa, o inclusive anularla... En este sentido hay que entender la máxima de los jurisconsultos, de que conventio omnis intelligitur rebus sic stantibus. ${ }^{9}$

Muchos juristas de la doctrina contemporánea del derecho internacional han aceptado (unos con más cautela que otros) la doctrina RSS. Autores como Bluntschli y Bynkershoek proporcionan importantes aportaciones para que sea admitida la legitimidad de la cláusula, convirtiéndola con el tiempo en una norma del derecho internacional.

Finalmente, el jurista español Poch de Caviedes ${ }^{10}$ señala que en la Convención de la Habana de 1928 (único instrumento convencional previo a la Convención de Viena, que existió sobre el derecho de los tratados) se da cabida a la Doctrina $R S S$ en el artículo 15:

Podrá igualmente declararse la caducidad de un tratado cuando éste sea permanente y de aplicación no continua, siempre que las causas que le dieron origen hayan desaparecido y pudiera lógicamente deducirse que no se presentarán en el futuro.

La parte contratante que alegare esta caducidad, al no obtener el asentimiento de la otra o de las otras, podrá acudir al arbitraje, sin cuyo fallo favorable y mientras éste no se dicte, continuará en vigor las obligaciones contraídas.

\section{CONVENCIÓN DE VIENA SOBRE EL DERECHO DE LOS TRATADOS, 1980}

La Convención de Viena sobre el Derecho de los Tratados, suscrita en mayo de 1969 y que entró en vigor al depositarse el 35o. instrumento

9 Gómez Robledo, Antonio, op. cit., nota 1, p. 88.

10 Poch de Caviedes, Antonio, De la clause rebus sic stantibus a la clause de revisión dans les conventions internacionales. Res des Cours, 1966. 
de adhesión, en enero de 1980, no reconoce de manera abierta la RSS. Sus doctrinarios trataron de evitar incluirla de manera tácita en el texto de la convención. Sin embargo, la afirmación de que una norma general de derecho internacional, de origen consuetudinario, hacia del cambio en las circunstancias causa de terminación de los tratados, solamente fue marginalmente combatida durante el proceso de codificación del instrumento. De acuerdo con Remiro, las aprehensiones surgieron después, con la concreción del tipo, la explicación tácita de los casos en que no operaba y la adopción de garantías procesales frente a posibles abusos de una interpretación unilateral. ${ }^{11}$

Finalmente, la Cláusula RSS quedó contemplada en el artículo 62 de la Convención de Viena, aunque su texto nunca la denomina como tal:

\section{Artículo 62}

1) Un cambio fundamental en las circunstancias ocurrido con respecto a las existentes en el momento de la celebración de un tratado y que no fue previsto por las partes, no podrá alegarse como causa para dar por terminado el tratado o retirarse de él, a menos que:

a) La existencia de esas circunstancias constituyera una base esencial del consentimiento de las partes en obligarse por el tratado; y

b) Ese cambio tenga por efecto modificar radicalmente el alcance de las obligaciones que todavía deban cumplirse en virtud del tratado.

2) Un cambio fundamental en las circunstancias no podrá alegarse como causa para dar por terminado un tratado o retirarse de él:

a) $\mathrm{Si}$ el tratado establece frontera; o

b) Si el cambio fundamental resulta de una violación, por la parte que lo alega, de una obligación nacida del tratado o de toda otra obligación internacional con respecto a cualquier otra parte del tratado.

3) Cuando, con arreglo a lo dispuesto en los párrafos precedentes, una de las partes pueda alegar un cambio fundamental en las circunstancias como causa para dar por terminado un tratado o para retirarse de él, podrá también alegar ese cambio como causa para suspender la aplicación del tratado. $^{12}$

Es importante señalar que para el caso que nos ocupa, el Tratado de Aguas Internacionales, la aplicación de la Cláusula RSS tal como se referencia en la Convención de Viena, no es aplicable, debido a que el trata-

11 Remiro Brotóns, Antonio, op. cit., nota 2, p. 485.

12 Convención de Viena sobre el Derecho de los Tratados, 1980, artículo 62. 
do se firma en 1944 y la convención entra en vigor hasta 1980. Ante este hecho, nuestra referencia se mantendrá en los clásicos del derecho internacional, y asumimos a la $R S S$ como un elemento fundamental y doctrinario del derecho internacional.

\section{LA APLICACIÓN DE LA CLÁUSULA REBUS SIC STANTIBUS}

Haremos nuevamente referencia al texto del maestro Gómez Robledo, en donde se señala que el primer caso de la historia diplomática en que un Estado esgrimió ante los demás la doctrina del cambio fundamental de circunstancia, fue la denuncia del Tratado de París de 1856. Una vez que Rusia perdió la guerra de Crimea, esa nación hubo de acceder a la neutralización del Mar Negro, obligándose a no mantener fuerzas navales ni construir fortificaciones en su litoral. Pero en 1870, en plena guerra franco-prusiana, el ministro de Relaciones Exteriores de Rusia envió una comunicación a las potencias firmantes del tratado, una resolución de su gobierno de exonerarse de los compromisos contraídos en razón de los cambios que en el intervalo habían tenido lugar en la situación internacional. El gobierno británico protestó de inmediato y convocó a una reunión en Londres en donde asistió prácticamente todo el concierto europeo. Rusia alegó que el cambio de circunstancias consistía fundamentalmente en que ahora tenía ante sí, no solamente a Turquía, sino a un estado de reciente creación: Rumania. Además, la técnica armamentista había progresado a tal manera que no era justo que Rusia se mantuviera indefensa en aquel mar y en aquel litoral. Después de esta reunión y con el consentimiento europeo, Rusia pudo librarse de esta restricción. ${ }^{13}$

Para terminar esta sección señalaremos que si bien la jurisprudencia internacional no llegó a aceptar un caso claro de aplicación de la $R S S$ antes de la Convención de Viena, una vez que aparece la referencia en dicho instrumento, la situación se ha modificado sustancialmente, ya que la cláusula ha pasado a ser derecho positivo. El maestro Gómez Robledo señala un caso aparecido en el Reporte de la Corte Internacional de Justicia de 1993, en el que se señala al respecto de un caso sobre pesquerías anglo-islandesas lo siguiente: 
El derecho internacional admite que si un cambio fundamental de las circunstancias que determinaron a las partes celebrar un tratado, transforma radicalmente el alcance de las obligaciones impuestas por el tratado, la parte afectada por dicho cambio puede, bajo ciertas condiciones, alegar la terminación o la suspensión del tratado. Este principio, así como las condiciones y excepciones a que se encuentra sujeto, han sido señaladas por el artículo 62 de la convención de Viena sobre el Derecho de los tratados, el cual puede ser considerado en muchos aspectos como una codificación del derecho consuetudinario existente en lo que concierne a la terminación de un tratado en relación con el cambio de circunstancia. ${ }^{14}$

\section{El TRATAdo SOBRE Distribución DE AGUAS INTERNACIONALES ENTRE LOS ESTADOS UNIDOS MEXICANOS Y LOS ESTADOS UNIDOS DE AMÉRICA}

A partir de la fecha en que se celebró el Tratado de Guadalupe Hidalgo en 1848 y el de "La Mesilla" en 1853 en los que se definieron los nuevos límites fronterizos entre México y EUA, ha iniciado el conflicto bilateral por la definición del cauce, el uso de los caudales y de los recursos hídricos de los tres ríos que se encuentran en esta región limítrofe: el Bravo, el Colorado y el Tijuana.

El uso de las aguas de estas corrientes para fines agrícolas, de producción, de desagüe y limítrofe ha dado origen a dos tipos de problemas básicos: uno jurídico, de muy amplio espectro y otro de ingeniería. Ambos se han traducido en situaciones de controversia diplomática bilateral hasta nuestros días. Dentro del ámbito jurídico se han tratado de definir y hacer valer los derechos de los países en el aprovechamiento de las aguas de estos ríos y la cuantificación de esos derechos; el segundo tipo de problemas se ha enfocado presentar propuestas técnicas y prácticas para la utilización de las aguas en cada país.

El conflicto permanente debido a las acciones realizadas en ambos lados de las cuencas, así como la profundización de las notas de protesta entre ambos gobiernos y una variedad de instrumentos jurídicos a los cuales apelar, volvió indispensable la elaboración y firma de un tratado bilateral que sentara las bases para el uso de las cuencas fronterizas entre México y EUA. 
El primer tratado de distribución de aguas entre los dos países fue la Convención para la Equitativa Distribución de las Aguas del Río Grande, mejor conocida como la Convención de 1906. Esta convención estipula la entrega de aguas del Río Bravo a México por parte de EUA. Para tal fin se construyeron obras de infraestructura y nuestro país aceptó que esta entrega de agua por parte de EUA no significaría el reconocimiento de ningún derecho por parte de México a dichas aguas, así como el retiro de cualquier reclamación a las aguas del Río Grande, entre la boca del actual Canal Mexican y Fort Quitman, Texas.

Después de cuatro décadas de firmar el primer tratado, influenciados por los acontecimientos de la Segunda Guerra Mundial y después de una serie de dificultades técnicas, políticas y diplomáticas, ambos países decidieron firmar un nuevo acuerdo sobre aguas internacionales, bajo el cual se dio forma a la actual Comisión Internacional de Límites y Aguas. De esta forma, en 1944 se firmó el Tratado sobre Distribución de Aguas Internacionales entre los Estados Unidos Mexicanos y los Estados Unidos de América.

El objeto del tratado fue "fijar y delimitar claramente los derechos de las dos repúblicas sobre los ríos Colorado y Tijuana y sobre el río Bravo (Grande, de Fort Quitman, Texas, EUA, al Golfo de México), a fin de obtener su utilización más completa y satisfactoria". ${ }^{15}$

\section{CARACTERÍSTICAS DEL TRATADO. PARTICULARIDADES DEL SUMINISTRO}

La comprensión del tratado requiere del recuento de una gran cantidad de información, antecedentes y el entendimiento de múltiples conflictos bilaterales al respecto. Pero en virtud del objetivo que nos ocupa en el presente artículo, es importante comprender las características hídricas predominantes en la época y las consideraciones que se hicieron en materia de ingeniería, volúmenes de agua y la forma de distribuirla para poder llegar al entendimiento y la firma.

De acuerdo con los estudios técnicos que en esa época se llevaron a cabo para determinar estas proporciones, el total del afluente del Río

15 Secretaría de Relaciones Exteriores, Oficina de Límites y Aguas Internacionales, "Preámbulo", El Tratado de Aguas Internacionales, México, 1947. 
Bravo era de 12,135 millones de metros cúbicos en promedio al año, de los cuales la cuenca ubicada en los EUA aportaba 6,325 millones de $\mathrm{m}^{3}$ (el 52.1\%), mientras que la cuenca mexicana aportaba al caudal 5,810 millones (el 47.9\%).

En virtud de que la aportación al caudal por parte de la cuenca mexicana era menor que la de EUA y con el fin de hacer equitativa la distribución y uso del agua, en el artículo 4o. del tratado se contempla la entrega de México a EUA de un volumen anual de 431.7 millones de $\mathrm{m}^{3}$ de agua, a través de ciertos tributarios específicos, en promedio de ciclos de cinco años.

El propio tratado establece las condiciones para cubrir los volúmenes deficitarios de las entregas de agua, señalando que en caso de extraordinaria sequía o de serio accidente en los sistemas hidráulicos de los afluentes mexicanos que haga difícil para México escurrir los 431.7 millones de metros cúbicos que se asignan a EUA, los faltantes que existieran al final del ciclo de cinco años se repondrán en el ciclo siguiente con agua procedente de los mismos tributarios.

Por otro lado, EUA se comprometió a enviar a los estados mexicanos de Baja California y Sonora, mil 831 millones de metros cúbicos provenientes del caudal del Río Colorado.

\section{LA SEQUÍA Y EL CONFLICTO INTERNACIONAL}

Con este mecanismo jurídico y el apoyo de la Comisión Internacional de Límites y Aguas, durante décadas, ambas naciones han logrado mantenerse en general satisfechas respecto a sus requerimientos de agua en la frontera, salvo eventuales situaciones que han requerido una atención más cuidadosa y que se han resuelto en su mayoría mediante actas firmadas por la comisión.

Sin embargo, se han presentado circunstancias que no se han podido resolver mediante este mecanismo, derivadas de la sequía que en los últimos años se ha presentado en la región. Esta situación, además de haber provocado un grave daño para el desarrollo de la zona, desencadenó una serie de situaciones adversas entre los estados ribereños, que incluso tuvo alcances internacionales y finalmente derivó en conflictos diplomáticos. 
El impacto de esta situación fue tal, que propició la participación de prácticamente todos los niveles de gobierno, incluyendo por supuesto una intensa intervención del Poder Legislativo.

El problema de la seguía y el retraso del pago por parte de México derivó en la firma del Acta 307 de la Comisión Internacional de Límites y Aguas. La firma de esta acta se dio en el marco de la primera reunión entre los presidentes de EU y México, en el rancho privado de este último. Fue el primer acuerdo de corto plazo en materia hídrica. Firmada el 16 de marzo de 2001, indica que:

Los comisionados tomaron nota de las conversaciones llevadas a cabo entre el presidente de México, Vicente Fox Quesada, y el presidente de Estados Unidos, George W. Bush, en San Cristóbal, Guanajuato, el 16 de febrero de 2001, donde se formuló una solicitud de agua por un volumen de 740 millones de metros cúbicos (600 mil acres pie) al 31 de julio de 2001, para reducir el déficit actual de las aportaciones de los tributarios mexicanos del río Bravo. En seguimiento a esta conversación, los comisionados revisaron los datos proporcionados por los ingenieros principales y observaron que, de finales de septiembre de 2000 al 3 de marzo de 2001, se ha contabilizado a favor de Estados Unidos un volumen de 287 millones de m3 (231,674 acres pie) por lo que sólo restaría cubrir, para el 31 de julio de 2001, un volumen de 453 millones de m3 (367,252 acres pie). ${ }^{16}$

La solicitud del gobierno estadounidense de la cual "tomaron nota" los comisionados fue realizada durante la guerra de EUA contra Irak, lo que dio un tono especial a los compromisos que demandaba aquella nación estadounidense, bajo el preámbulo de que:

Los gobiernos de México y de Estados Unidos, animados por el espíritu de amistad que prevalece en la relación entre los dos países y comprometidos a prevenir que tengan que encontrarse nuevamente en situaciones como las que aquí se han tratado, trabajan conjuntamente para identificar medidas de cooperación en materia de manejo de sequías y de manejo sustentable de esta cuenca. ${ }^{17}$

16 Cila, Acta 307 "Cobertura parcial del déficit en la aportación de los tributarios mexicanos del río Bravo entre Fort Quitman y la presa Falcón”, Washington, 16 de marzo de 2001. Disponible en: www.sre.gob.mx/cila.

17 Idem. 
De acuerdo con Delgado Ramos, ${ }^{18}$ el trasfondo del Acta 307 es doble. Por un lado, fue una negociación que jugó un papel fundamental en el preámbulo de las elecciones a gobernador de Texas. Por el otro lado asentaba legalmente la posibilidad de violar las fechas de pago — de México a EUA - en el sentido de su adelanto, algo urgente, pues la severa sequía ya comenzaba a afectar seriamente a los agricultores texanos al comprometer el riego de los cultivos de la temporada de verano.

Con base en el Tratado de 1944, México debe pagar anualmente un monto mínimo garantizado de agua que no es reducido proporcionalmente en caso de intensa sequía (algo que sí sucede en el caso de los pagos de agua de EUA en el Colorado y en el tramo del Bravo que va de El Paso a Fort Quitman). Por otro lado, el pago mexicano de agua puede hacerse en un ciclo de cinco años de tolerancia, por lo que México, en este caso, no estaba obligado a pagar su adeudo. En el caso del ciclo 25 (1992-1997), que venció en septiembre de 2002, ya estaban pagadas las cuotas correspondientes antes de que se firmara el Acta 307, mientras que el pago por el ciclo 26 podía ser cubierto hasta 2007.

Lo anterior hace pensar que las fechas de vencimiento fueron "manipuladas" de tal forma que se hizo creer a la opinión pública, particularmente a la de EUA, que México se estaba "robando" el agua. Esta situación dio lugar a que los gobernadores estadounidenses de la región fronteriza hicieran serías demandas al gobierno de México para que pagara el agua que seguía adeudando, llegando inclusive a presentar amenazas de investigación sobre la disponibilidad y uso del agua en territorio mexicano.

Como señala Delgado Ramos, las presiones para que se efectuaran los pagos de agua se llevaron a tal nivel que con el ánimo de cumplir el compromiso internacional no se consideró el alto costo que ello representó para los agricultores mexicanos. Con el fin de comprometer más al país al pago adelantado de agua, se negociaron las actas 308 y 309. La primera señalaba que la Comisión Internacional de Límites y Aguas contabilizará a favor de EUA $111 \mathrm{mil} \mathrm{m}^{3}$ (90,000 a. p.) de las aguas asignadas a México en las presas internacionales de la Amistad y Falcon, de conformidad con los entendimientos referidos en el apartado "A" de esta acta, mientras que la segunda establecía que México deberá entregar el agua que se 2005. 
ahorre con obras de modernización y tecnificación de infraestructura hidráulica, modificando el monto y procedencia del mínimo del líquido a pagar anualmente, especificando que se debe transferir de inmediato el agua almacenada que rebase los 1,044 millones de $\mathrm{m}^{3}$.

La reacción de gobernadores y legisladores de diversas fracciones parlamentarios fue enérgica ante esta situación. El senador de Tamaulipas, Homero Díaz Rodríguez, señalaba que estas actas significaban una franca violación al Tratado Internacional de Límites y Aguas, ya que se privaba de dicho líquido vital tanto a las ciudades fronterizas como al campo de Tamaulipas... "no basta informar al Senado para modificar los términos de un tratado internacional, sino hay que solicitar que se dictamine y apruebe la modificación". ${ }^{19}$

Por su parte, el senador Demetrio Sodi, entonces del Grupo Parlamentario del PRD, presentó un Punto de Acuerdo para proponer una controversia constitucional por la suscripción del Acta 308. De acuerdo al Senador Sodi, el:

Convenio o acuerdo contenido en la minuta 308, adiciona, anexa, introduce aspectos totalmente nuevos que no estaban contemplados antes en el Tratado de Distribución de Aguas Internacionales de los Ríos Colorado, Tijuana y Bravo, en consecuencia con ello se modifica sustancialmente el tratado internacional en comento y se invade la esfera de competencia exclusiva del senado de la república, pues se está pagando nuevamente a Estados Unidos en una forma diversa que no está contemplada en el Tratado Internacional de referencia.

Cabe señalar que los volúmenes de agua no contemplados en el tratado internacional referido, pagados a Estados Unidos a través de la Minuta 307 y 308 , pertenecen a los usuarios del distrito de riego 025 , a quienes se ha privado por dos años consecutivos del agua a que tienen derecho de acuerdo a los títulos de concesión que tienen vigentes. Ocasionándose así, cuantiosos daños y perjuicios a los usuarios y, un problema social de gran magnitud, pues se han afectado a 14,500 familias de agricultores quienes cultivan 202,000 hectáreas de riego en el distrito.

19 Klérigan, Efraín y Domínguez, Miguel, "Son ilegales las minutas de CNA: legisladores", El Norte, 8 de octubre de 2003. 
De ello, se desprende que los preceptos constitucionales violados lo constituyen los artículos 76 fracción I, 89 fracciones I y X, 133, 79 f) y 49 de nuestra Constitución Política Federal. ${ }^{20}$

Por otra parte, el diputado Diego Cobo Terrazas del PVEM se ha manifestado por la revisión del acuerdo bilateral de 1944, señalando que las condiciones ambientales e hidrológicas que se observan en la actualidad son radicalmente distintas a las de hace 60 años.

Durante 2002, en los senadores de oposición reinaba la conciencia de que en los estados de la franja fronteriza se sufría una situación crítica tras varios años ininterrumpidos de sequía, lo cual mantenía a las presas en apenas diez por ciento de su capacidad de almacenamiento. Se aceptaba que EUA tenía el derecho de reclamar el agua, pero siguiendo el espíritu del tratado, debía comprenderse la indisponibilidad del recurso y abstenerse de cualquier tipo de presiones políticas, como las que estaba ejerciendo el gobernador de Texas, en función de intereses estrictamente electorales.

\section{CONDICIONES HIDROLÓGICAS Y CAMBIO CLIMÁTICO. UN CAMBIO EN LAS CIRCUNSTANCIAS}

El efecto de las sequías en México se ha hecho cada vez más drástico en las últimas décadas. Los estudios con los que contaba el grupo de expertos que negoció las condiciones técnicas para la asignación de responsabilidades en el tratado, no podía suponer la modificación que se llevaría a cabo en el clima y en el ciclo de las sequías. De acuerdo con datos de la Comisión Nacional del Agua, el grado de afectación de los cuatro grandes periodos de sequía ocurridos durante el siglo XX se ha ido incrementando en forma drástica. Durante la última gran sequía ocurrida de 1993 a 1996, la superficie que sufrió una afectación severa fue prácticamente el equivalente a la mitad del territorio nacional, con efectos mucho más drásticos en las estados del norte. Esta situación fue prácticamente imposible de prever en 1944 (véase gráfica 1 en la siguiente página).

20 Los tres párrafos constituyen una cita textual de la versión electrónica de Senado de la República, Gaceta Parlamentaria. Las líneas resaltadas con cursivas corresponden a las mayúsculas negritas del Punto de Acuerdo. Véase: http://www.senado.gob.mx/content/sp/sp/content/gaceta/content/permanente/58/content/31/Proposicion_Sodi_Controve rsia.html. 
Gráfica 1. Grado de afectación de las cuatro grandes sequías del siglo XX

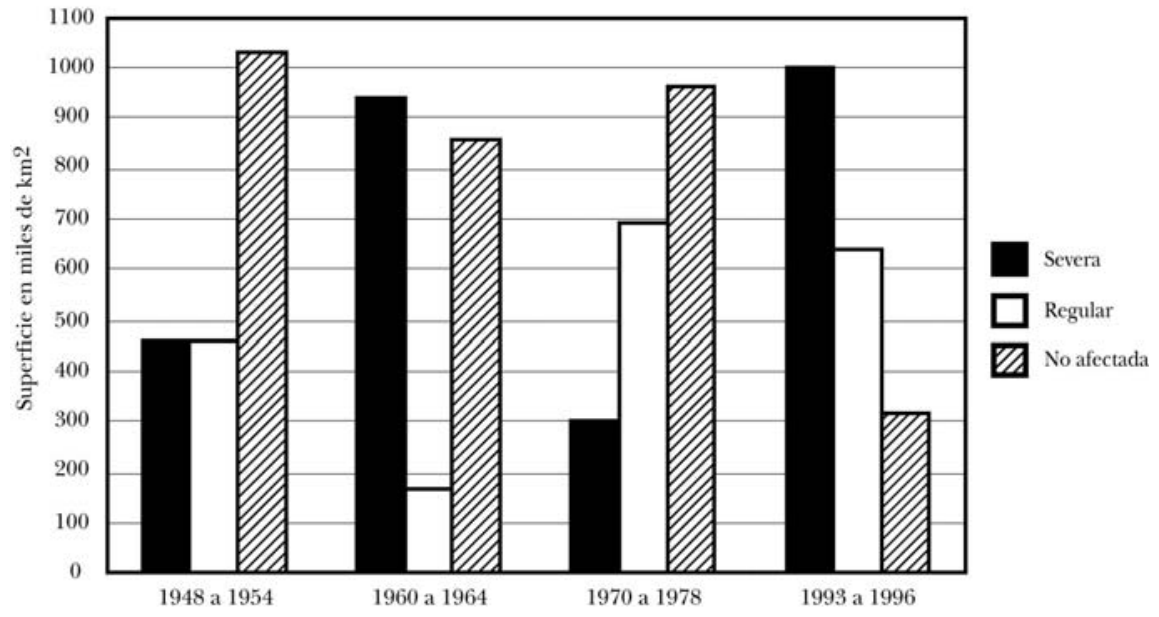

FUENTE: Elaboración propia con base en datos de la Comisión Nacional del Agua. 
Por otra parte, el cambio climático evidentemente ha modificado el caudal y las afluentes, el escurrimiento y la evaporación de agua en los ríos a los que hace mención en el tratado (véase gráfica 2 en la siguiente página).

\section{CONCLUSIÓN. VIABILIDAD DE LA APLICACIÓN \\ DE LA ClÁUSUla REBUS SIC STANTIBUS EN EL TRATADO \\ DE AGUAS INTERNACIONALES}

Los indicadores mostrados en el apartado anterior nos invitan a reflexionar sobre el cambio drástico de la situación en las vertientes de los sistemas hídricos fronterizos y se hace indispensable una serie de estudios técnicos que demuestren que las condiciones imperantes en las cuencas de los ríos Bravo, Tijuana y Colorado han cambiado sustancialmente con relación a lo que se estipuló en El Tratado de 1944. Estos cambios han llevado a firmar actas que en opinión de varios legisladores, están modificando en forma substancial el contenido original de este instrumento internacional.

Por lo tanto, mientras se sientan las bases para negociar un nuevo tratado que refleje las condiciones actuales que se vive en la región fronteriza entre México y EUA, y después de haber analizado algunos de los principios y doctrinas de derecho internacional, consideramos que sí existe la posibilidad de apelar a la Cláusula $R S S$ en virtud de que existe un cambio fundamental, tanto técnico como jurídico, de las condiciones en que se originó el tratado que aquí se comenta.

Para ello será necesario un trabajo conjunto, tanto a nivel técnico como jurídico, en donde la Sección Mexicana de la Comisión de Límites y Aguas exponga su punto de vista y señale los motivos, tanto en el ámbito de ingeniería, como en el componente legal, que le motivaron para la elaboración y firma de las actas que hemos señalado.

Este trabajo conjunto podría arrojar los elementos necesarios para poder, en primera instancia, montar un elemento de presión sólido ante los EUA que permitiera la negociación de un nuevo instrumento en el que se incluyera todos los avances tecnológicos para determinar una distribución de agua más equitativa, dejando de lado la asignación de cuotas estáticas y rígidas, ya que hemos visto que nos enfrentamos a un entorno cambiante. Por otro lado, queda la posibilidad de apelar a la Corte 
Gráfica 2. Cambio de la temperatura global en el mundo

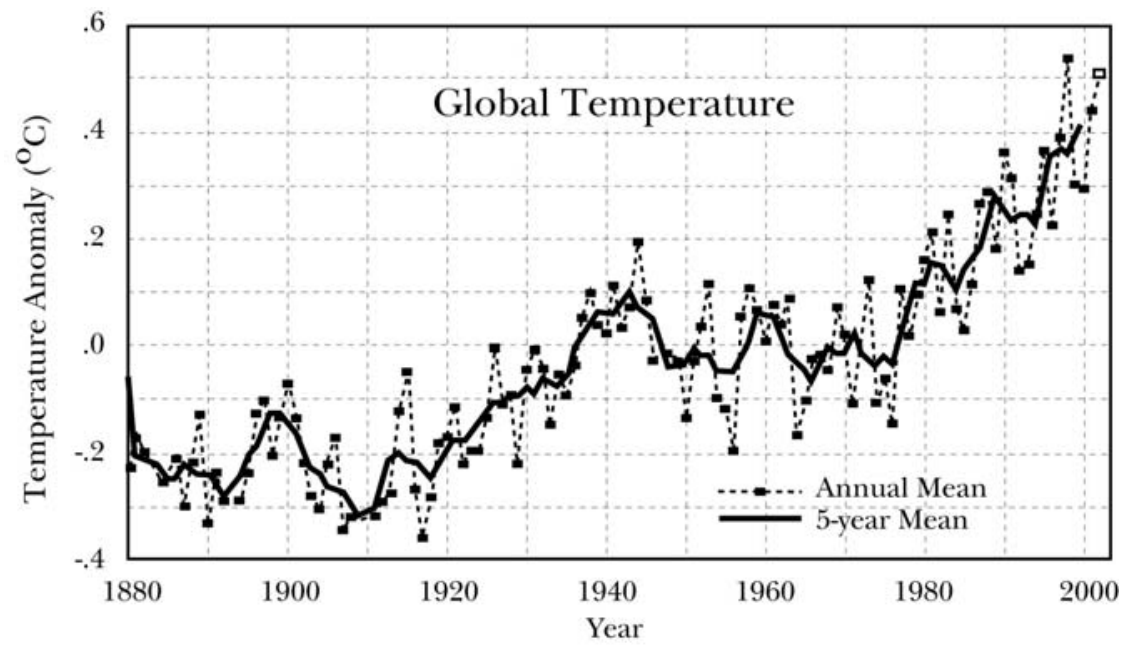

FUENTE: Tomado de la Conferencia sobre Cambio Climático de la World Future Society, febrero de 2006. 
Internacional de Justicia, si tomamos como base la doctrina del derecho internacional y tenemos presente una sentencia de la propia corte:

El derecho internacional admite que si un cambio fundamental de las circunstancias que determinaron a las partes celebrar un tratado, transforma radicalmente el alcance de las obligaciones impuestas por el tratado, la parte afectada por dicho cambio puede, bajo ciertas condiciones, alegar la terminación o la suspensión del tratado. Este principio, así como las condiciones y excepciones a que se encuentra sujeto, han sido señaladas por el artículo 62 de la Convención de Viena sobre el Derecho de los Tratados, el cual puede ser considerado en muchos aspectos como una codificación del derecho consuetudinario existente en lo que concierne a la terminación de un tratado en relación con el cambio de circunstancia. ${ }^{21}$

21 Traducción mía de una sentencia, emitida por la Corte Internacional de Justicia, que tomé de http://www.icj-cij.org/icjwww/idecisions.htm. 